\title{
L'ENTR'AIDE ET LA GROIX-ROUGE
}

Lorsqu'en 1863, les Etats chrétiens tinrent conseil à Genève et jugèrent opportun d'adresser au monde entier un appel retentissant en faveur des militaires blessés, un grand pas en avant fut fait dans la voie de la civilisation. Des légions de volontaires surgirent prêts à affronter les plus grands dangers pour combler une lacune regrettable dans les institutions sociales de ce temps. Il suffit au christianisme de faire appel à la charité que doivent professer ses adeptes pour les voir s'enrôler en foule sous son drapeau.

Le succès dépassa même les espérances des promoteurs de ce mouvement. Cie ne fut pas sans surprise que l'on vit la Turquie, la Perse, ainsi que la Chine, le Siam, la Corée et le Japon accéder successivement à la Convention de Genève. Ce qui n'avait été d'abord qu'une manifestation de solidarité chrétienne s'éleva au rang de solidarité humaine. Bien que l'intérêt matériel des signataires ne leur en fît en aucune façon une obligation, nombre d'Etats s'unirent sans distinction de race ni de religion dans un même sentiment de sympathie en face de la souffrance individuelle. Quel fut le mobile qui les fit agir ainsi? Quelle puissance, plus haute que l'intérêt, plus universelle que les religions, réussit à réunir en un faisceau toutes ces volontés, si divergentes à tant d'égards? Ne serait-ce pas ce que Kropotkine, dans un livre récent, a nommé l'Entr'aide? ${ }^{1}$

On a beaucoup parlé dans ces cinquante dernières années de la lutte pour la vie. Après Darwin et Spencer, nombreux furent les hommes de science, sociologues, philosophes ou romanciers qui mirent au premier rang des facteurs de l'évolution, la compétition, la sélection par survivance du plus apte, et, en fait, l'égoïsme et l'arrivisme sous leurs formes les plus basses. Kropotkine s'élève

1 Pierre Kropotkine, l'Entr'aide, un facteur de l'évolution, traduit de l'anglais par L. Bréal (Hachette 1906). 
contre cette conception du progrès et lui oppose le sentiment de la solidarité qu'il nomme l'entr'aide. La lutte reste le fait primordial, mais non plus au premier plan; l'union pour la lutte, le secours mutuel entre individus d'une même espèce et parfois même d'espèces différentes, devient le facteur principal de l'évolution.

Ce fut, d'ailleurs, l'opinion de Darwin lui-même, ainsi que l'atteste Bates, qui fut longtemps son collaborateur. Mais, avarit de montrer les effets de l'union pour la lutte, Darwin eut à mettre d'abord en lumière le ròle de la lutte elle-même comme moyen de sélection. Ses successeurs, au lieu de continuer son idée en l'élargissant, la restreignirent au contraire, et, prenant l'expression de la lutte pour la vie dans son sens étroit, s'en emparèrent pour faire l'apogie de l'individualisme.

Kropotkine répare donc une injustice faite à la mémoire de Darwin quand, s'attachant à l'idée primitive du grand naturaliste anglais, il expose le rôle prépondérant de l'entr'aide dans l'évolution des espèces animales.

Mais il ne s'arrête pas là. En vertu d'une étroite parenté entre les animaux et les hommes, il envisage le facteur de la sélection dans l'espèce humaine, et, partant des peuples sauvages les plus primitifs, il s'élève graduellement dans l'échelle de la civilisation jusqu'à notre époque de haute différenciation sociale.

Selon lui, le vrai facteur de l'évolution fut donc l'entr'aide. Kropotkine montre que la sélection amène invariablement le triomphe des éléments les plus sociables, de ceux chez qui le secours mutuel est le plus développé.

* Dans la grande lutte pour la vie, dit-il, - pour la plus grande plénitude et la plus grande intensité de vie, avec la moindre perte d'énergie - la sélection naturelle cherche toujours les moyens d'éviter la compétition. Les espèces animales dans lesquelles la lutte individuelle a été réduite à ses plus étroites limites el où l'habitude de l'entr'aide a atteint le plus grand développement, sont invariablement les plus nombreuses, les plus prospères et les plus ouvertes au progrès. La protection mutuelle obtenue de cette façon, la possibilité d'atteindre à un âge avancé et d'accumuler de l'expérience, un état intellectuel plus avancé, et le développement 
d'habitudes de plus en plus sociables, assurent la conservation de l'espèce, son extension et son évalution progressive. Les espèces non sociables, au contraire, sont condamnées à dépérir. 刃. La survivance des êtres chez lesquels le sentiment de solidarité était le plus vivace, s'accentua nécessairement de génération en génération. L'atavisme aidant, ce fut bientôt chez les animaux un instinct des plus vivaces.

Mais l'auteur va plus loin encore. Ses chapitres sur le monde animal ne sont qu'une introduction zoologique à un ouvrage social.

Des animaux, Kropotkine passe à l'homme. Il retrouve ici l'entraide comme premier soutien dans la lutte pour l'existence. Que nous sommes loin de la guerre de tous contre tous, que Hobbes représente comme l'état naturel de l'homme! Huxley, paraphrasant Rousseau, déclare: « Les premiers hommes qui substituèrent la paix mutuelle à la guerre mutuelle - quel que soit le motif qui les força à faire ce progrès - créèrent la société. 》 - « La société n'a pas été créée par l'homme, ajoute très justement notre auteur, elle est antérieure à l'homme. L'individualisme effréné est une production moderne et non une caractéristique de l'humanité primitive. )

Pour appuyer sa thèse, il examine les récits des premiers missionnaires et colons qui entrèrent en contact avec les races primitives. Tous sont d'accord pour louer la morale élevée de ces peuples, et leur attachement à leur clan, qui est pour eux une chose plus importante que leur vie individuelle.

De l'ethnologie, l'auteur passe à l'archéologie ou plutòt à l'authropologie préhistorique. Il établit un parallèle entre l'homme de l'âge de la pierre et le sauvage de notre époque. Puis il parle des barbares, et suit pas à pas, à travers le moyen-âge, l'évolution du sentiment de solidarité. Il est obligé pour cela de lire pour ainsi dire entre les lignes de l'histoire, qui jusqu'ici a donné un relief exclusif aux guerres et aux actions d'éclat de quelques grandes individualités. Mais, en y regardant de près, il est facile de reconstituer la civilisation intime des périodes historiques, et l'on voit l'association et la coopération y jouer toujours un rôle prépondérant. 
De nos jours, avec l'âpreté grandissante de la lutte, les manifestations de solidarité sont aussi devennes plus nombreuses. Les populations misérables des grandes villes, ne pourraient subsister sans le soutien mutuel. Même dans d'autre classes sociales, si l'on pouvait évaluer ce qu'il se consacre de temps et d'argent à l'entr'aide sous mille forme différentes, on serait certainement stupéfait. L'auteur parle des associations ouvrières, des différentes sortes de biens communaux, des mutualités, des sociétés coopératives, des syndicats agricoles, voire des clubs sportifs. Il est regrettable qu'il aît omis quelques-unes des formes les plus élevées de l'association, entre autres l'œuvre des secours aux blessés de la guerre, l'une des plus hautes manifestations de l'instinct de cet "entr'aide " qui fait le sujet de son livre.

Si l'on pouvait, en regard de l'évolution, évaluer la puissance respective de la lutte pour la vie et de l'entr'aide, laquelle l'emporterait? Les livres de l'histoire, les chroniques quotidiennes des journaux, les spéculations des individualistes montrent surtout la lutte. «Par contre le facteur de l'entr'aide n'a reçu jusqu'à présent aucune attention. Les écrivains de la génération présente et passée le nient purement et simplement, ou même le tournent en dérision ». N'était-il pas juste de lui rendre la place qui lui est due?

Kropotkine termine son ouvrage par quelques considérations d'une grande élévation de pensée. Il relève ce fait que toutes les révolutions morales ont été un retour au sentiment de solidarité humaine. S'il avait voulu développer cette thèse, il aurait trouvé dans les vieux livres de l'Inde ou de la Chine - particulièrement dans le merveilleux Tao-te-king du moraliste chinois Lao-tse, ainsi que dans le Coran, des préceptes remarquables touchant l'entr'aide.

La conception plus élevée qui nous dit: «Point de vengeance pour les injures » et qui nous conseille de donner plus que l'on n'attend de recevoir de ses voisins, est proclamée comme le vrai principe de la morale, principe supérieur à la simple notion d'équivalence, d'équité ou de justice, et conduisant à plus de bonheur. Un appel est fait ainsi à l'homme de se guider, non seulement par l'amour, qui est toujours personnel et s'étend tout au plus à la tribu, mais par la conscience de ne faire qu'un avec tous les êtres humains. 
Il résulte de l'ensemble des documents réunis par Kropotkine, que le sentiment de l'entr'aide fut inné en l'homme bien avant notre époque de civilisation avancée et qu'il le tient, par atavisme, des temps les plus reculés. Ce que l'on a su ajouter de nos jours à la solidarité primitive des premières agglomérations sociales, c'est l'organisation savante dans les secours à apporter à ceux qui en ont besoin, c'est l'application du principe de la division du travail dans le domaine de la charité. L'entr'aide étant humaine avant tout, et de ce fait universelle, elle est de toutes les religions, de tous les peuples. Son esprit anime ceux qui se réunissent dans le but de secourir les blessés des champs de bataille, comme cenx qui s'associent pour une œuvre commune de secours mutuel contre les souffrances et les risques de la lutte journalière pour la vie.

L'entr'aide, c'est la notion de l'homme frère, c'est la sympathie à l'égard de tous ceux qui sonffrent. Sans doute le sentiment religieux contribue à stimuler cet instinct de solidarité humaine mais à cet égard toutes les religions sont d'accord et unies dans un but commun. Et voici réalisée la paix des religions sur le terrain, inné en l'homme, de l'entr'aide universelle.

Adolphe FERrière, licencié ès sciences sociales.

DERNIÈRES PUBLICATIONS DU COMITÉ INTERNATIONAL

La liste des publications du Comité international compte déjà trois éditions. - La dernière élant de janvier 1900, c'est-à-dire vieille de six ans, il peut être intéressant pour nos lecteurs de voir compléter cette liste par l'indication des publications parues dans ces dernières années. - C'est là ce qu'ils trouveront ci-dessous.

De la revision de la Convention de Genève. Etude historique et critique par M. G. Moynier. - Genève, 1898,

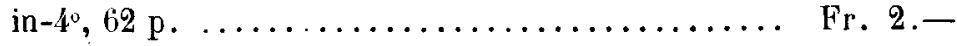

